\title{
Human Identification by Spatio-Temporal Symmetry
}

\author{
James B. Hayfron-Acquah, Mark S. Nixon, John N. Carter \\ Image, Speech and Intelligent Systems Group, Department of Electronics and Computer Science, \\ University of Southampton, Southampton S017 1BJ, United Kingdom. \\ \{jbha99r|msn|jnc\}@ecs.soton.ac.uk
}

\begin{abstract}
We describe spatio-temporal symmetry and its extraction via a Generalised Symmetry Operator. Its use in gait recognition is reinforced by the view from psychology that human gait is a symmetrical pattern of motion. We show that by including temporal information in our symmetry calculations we are not recognizing people by their body shape but also by their motion. Here, the new technique is applied to a database of 28 subjects, which equals in size the largest contemporaneous gait databases. The results of the new approach agree with earlier results that the symmetrical properties of human gait appear to be unique and can indeed be used for analysis and for recognition. The results achieved so far give promising performance and higher recognition rates than those of an earlier spatial approach. Performance analyses suggest that symmetry enjoys practical advantages such as ability to handle noise and occlusion, and especially when resolution is too low for other biometrics to be deployed.
\end{abstract}

\section{1: Introduction}

We present a new method of extracting spatio-temporal symmetry using the Generalised Symmetry Operator, applied in gait recognition. As a biometric, gait deals with the recognition of people by the way they walk regardless of the clothes worn or the differing background in image sequences. Notable advantages of gait over the other biometrics include lack of physical contact. Also, it is currently the only biometric at a distance when other biometrics may be of too low resolution, or obscured.

One motivation for gait recognition can be a real application scenario involving armed robbers. They usually put on helmets, masks, spectacles and gloves thus making it virtually impossible to use any other biometric such as the fingerprint or face. However, aiming not to attract attention by walking suspiciously or impeding their own gaits, the robbers will have to walk naturally thus exposing their own normal gait. Though it could be argued that physical condition such as drunkenness, pregnancy and injuries involving joints can affect an individual's motion, these factors are similar in principle to factors affecting other biometrics. There have been allied studies of gait [5], notable amongst these are medical studies, psychological studies, modelling human motion and tracking people. Amongst these, psychologists suggest gait is a symmetrical pattern of motion [3] and that humans perceive gait as unique.

Gait is perhaps the most recent biometric. The earliest approach was the spatio-temporal approach where gait signatures were derived from the spatio-temporal patterns of walking persons [6]. The gait signature was derived by normalising the fit of a 5-stick model to the spatiotemporal pattern and encouraging (85\%) recognition rates were achieved. Popularity of eigenspace approaches [5] is reflected in a recent approach [1] where PCA is applied to generate eigengaits. Supervised clustering then groups the training set into different classes. Similarity plots are then assigned to the various eigengaits for recognition, obtaining a recognition rate of $93 \%$ on a database of 6 subjects. Johnson et al presented a multi-view gait recognition method using recovered static body parameters (activity-specific biometrics) of subjects [11]. The technique uses the action of walking across multiple views to extract relative body parameters of subjects. The motion-capture data were used to adjust vision data of different views to a common reference frame. The approach gave a good result on a small database of subjects.

Shutler et al extended statistical gait recognition via temporal moments [10]. This derived statistics with an intimate relationship to gait, with symmetry properties. In [2], gait signatures were derived from the frequency components of the variations in the inclination of the human thigh. As pendula modelled the periodic motion of the thigh during walking, this again suggests that symmetry analysis is suited to gait recognition. As such, and coupled with medical studies [9] there is support for the contention that symmetry of gait can be examined. Earlier, we sought to deploy spatial symmetry analysis to sequences of people walking, and to good effect. We now describe its extension to spatio-temporal symmetry, to improve performance still further by including time in the symmetry calculations. Later we 
show how it can handle low resolution imagery, when other biometrics are obscured.

\section{2: Symmetry Extraction}

In [4], spatial symmetry extraction was calculated from sequences as follows. Feature templates are extracted from gait sequences to give template sequences. This was done by first removing the image background from each image frame in a sequence to obtain its corresponding image silhouette. The (Sobel) edge map of each image frame is then derived. The symmetry operator [8] uses each edge map to assign symmetry magnitude and orientation to image points by looking at the symmetry contributions from pairs of image points, accumulated at the midpoint of each pair of points. The result is the symmetry map. Fig. 1 shows the symmetry contribution of two edge points $P_{i}$ and $P_{j}$.

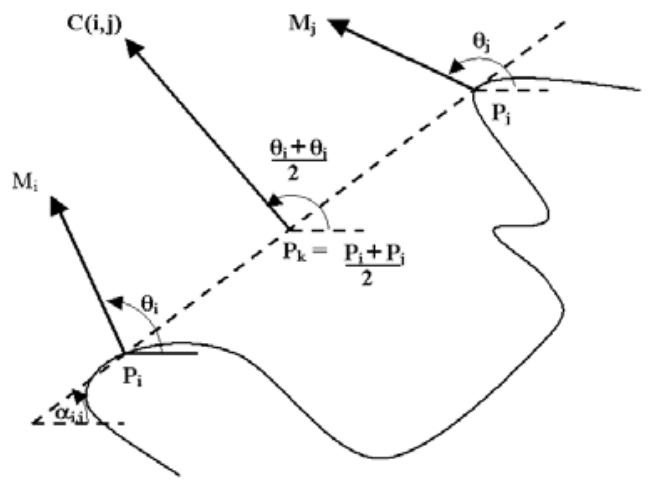

Fig. 1: Symmetry contribution calculation

The symmetry contribution, $C(i, j)$ between the points $P_{i}$ and $P_{j}$ is

$$
C(i, j)=D_{i, j} P h_{i, j} I_{i} I_{j}
$$

where $D_{i, j}$ and $P h_{i, j}$ are respectively the symmetry distance and the phase between the two points. $I_{i}$ and $I_{j}$ relate to intensity at points $P_{i}$ and $P_{j}$, respectively.

The symmetry distance is calculated from the symmetry distance weighting function. This reflects the distance between two different points $P_{i}$ and $P_{j}$, and is calculated as:

$$
D_{i, j}=1 / \sqrt{2 \pi \sigma} \exp \left(-\left(\frac{\left\|P_{i}-P_{j}\right\|-\mu}{2 \sigma}\right)\right), \forall i \neq j
$$

where $\sigma$ controls the scope of the function. Each value of $\sigma$ implies a different scale thus making it suited to multi-resolution schemes. A large value of $\sigma$ implies large-scale symmetry that gives distant points similar weighting to close points. Alternatively, a small value of $\sigma$ implies local operation and local symmetry. Recently the focus, $\mu$, was therefore introduced into the distance weighting function to control the focusing capability of the function [7], hence further improving the scaling possibilities of the symmetry distance function. The addition of the focus into the distance weighting function moves the attention of the symmetry operator from points close together to a selected distance.

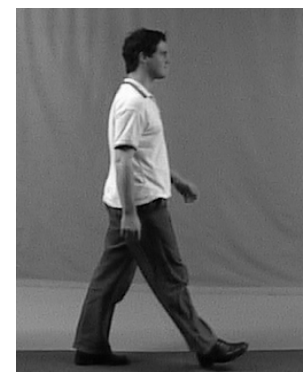

(a) original image

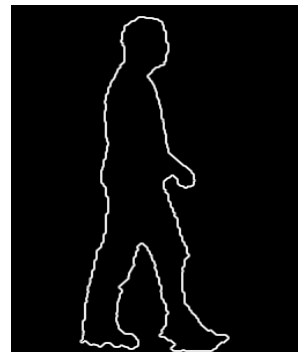

(c) detected edges

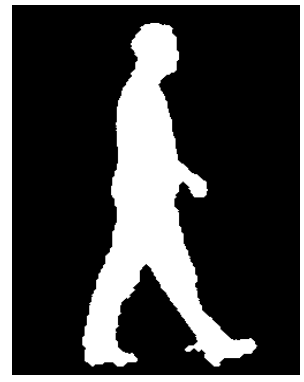

(b) silhouette

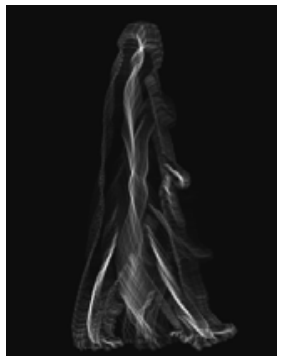

(d) spatial symmetry map
Fig. 2: Generating Spatial Symmetry

The logarithm, $I_{i}$, of the edge magnitude $M$ at point $i$ is $I_{i}=\log \left(1+M_{i}\right)$. Using the logarithm reduces the sensitivity to very strong edges. The phase weighting function between points $P_{i}$ and $P_{j}$ is:

$$
\begin{gathered}
P h_{i, j}=\left(1-\cos \left(\theta_{i}+\theta_{j}-2 \alpha_{i, j}\right)\right)\left(1-\cos \left(\theta_{i}-\theta_{j}\right)\right) \text {, } \\
\text { where } \quad \alpha(i, j)=\operatorname{atan}\left(\frac{y_{i}-y_{j}}{x_{i}-x_{j}}\right)
\end{gathered}
$$

is the angle between the line joining the two points and the horizon. The symmetry contribution is then midway between the two points. The total symmetry magnitude $M(p)$ of each point $p$ in the symmetry map is the sum of the contributions for all pairs of edge points $i$ and $j$ having their midpoint at $p$. Thus

$$
M\left(p_{k}\right)=\sum_{(i, j) \in p_{k}} C(i, j)
$$

Spatial symmetry detection is illustrated in Fig.2 showing increased symmetry relationship between the legs, below a central vertical-symmetry axis that arises from the symmetry of the torso.

Considering the distance between points that are separated spatially (within an image) and temporally (between images) to be components of a Euclidean distance metric gives as a new weighting function. Fig. 3 shows two images (one $T_{j, t}$ displaced $q$ frames from $T_{j, t-q}$, where $T_{j, t}$ and $T_{j, t-q}$ are not necessarily at the same spatial position) and their spatio-temporal symmetry map, which shows a distribution now centred more around the moving arms and legs, those parts more associated with gait. 


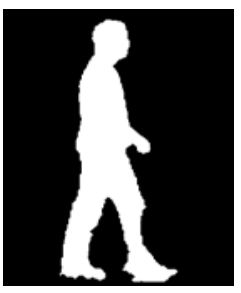

$T_{j, t-q}$

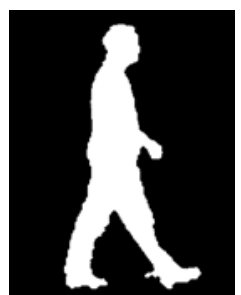

$T_{i, t}$

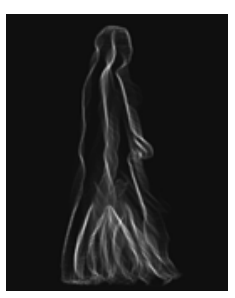

symmetry map
Fig. 3: Generating Spatio-Temporal Symmetry

The distance metric is the symmetry distance between two points separated in time and in space and can be used to replace the spatial symmetry weighting distance $D$, described earlier. This gives

$$
\left\|T_{i, t}-T_{j, t-q}\right\|=\sqrt{\left\|T_{j, t}-T_{j, t-q}\right\|^{2}+\left\|T_{i, t}-T_{j, t}\right\|^{2} / W}
$$

and for time/space weighting $W=1$, from Eqn. 2 the new distance is

$$
\left\|T_{i, t}-T_{j, t-q}\right\|=\begin{aligned}
& 1 / \sqrt{2 \pi \sigma_{s}} \exp \left(-\left(\frac{\left\|T_{j, t}-T_{j, t-q}\right\|-\mu_{s}}{2 \sigma_{s}}\right)\right)+ \\
& 1 / \sqrt{2 \pi \sigma_{t}} \exp \left(-\left(\frac{\left\|T_{i, t}-T_{j, t}\right\|-\mu_{t}}{2 \sigma_{t}}\right)\right)
\end{aligned}
$$

Here, we assume variance and focus are the same for time and space $\sigma_{s}=\sigma_{t}=\sigma$ and $\mu_{s}=\mu_{t}=\mu$, and $q=1$, so

$$
\left\|T_{i, t}-T_{j, t-1}\right\|=1 / \sqrt{2 \pi \sigma}\left[\begin{array}{c}
\exp \left(-\left(\frac{\left\|T_{j, t}-T_{j, t-1}\right\|-\mu}{2 \sigma}\right)\right)+ \\
\exp \left(-\left(\frac{\left\|T_{i, t}-T_{j, t}\right\|-\mu}{2 \sigma}\right)\right)
\end{array}\right]
$$

This replaces the distance metric $D_{i, j}$ in Eqn. 1 whilst the phase relationship remains unchanged. As such, we evaluate the symmetry relationship in time and in space. Note that all frames in a sequence are used. For this work each sequence of image frames consists of one gait cycle taken between successive heel strikes of the same foot, thus normalising for speed.

\section{3: Recognition Procedure}

Having obtained the symmetry maps for a given image sequence, we then derive the gait signature, $G S$ by averaging all the symmetry maps, $S_{j}$, as in [4], that is

$$
G S=\left(\sum_{j=1}^{N} S_{j}\right) / N
$$

where $N$ is the number of symmetry maps in a sequence. For purposes of comparing our new approach and that reported in [4], both approaches were applied to the same database. We used the same values of $\sigma$ and $\mu$ as earlier. First, the Fourier transform is applied to each of the gait signatures to give the description $F D$

$$
F D(u, v)=\sum_{N} \sum_{N} G S(x, y) e^{-j \frac{2 \pi}{N}(u x+v y)}
$$

where $G S$ is the gait signature. The $F D$ is then low-pass filtered to reduce sensitivity to high-frequency components by selecting only the descriptions within a circle of radius, $R$, as

$$
F D^{\prime}(u, v)=\left\{\begin{array}{ccc}
F D(u, v) & \text { if } & \left(u^{2}+v^{2}\right) \leq R^{2} \\
0 & \text { otherwise }
\end{array}\right.
$$

where the elements of $F D$ are the Fourier descriptions of the gait signatures after the low pass filtering. We used only the magnitude spectra here.

\begin{tabular}{|c|c|c|c|c|}
\hline \multirow{2}{*}{ Approach } & \multirow{2}{*}{$\begin{array}{c}\text { No. of } \\
\text { subjects }\end{array}$} & No. of & \multicolumn{2}{|c|}{ CCR (\%) } \\
\cline { 4 - 5 } & sequences & $k=1$ & $k=3$ \\
\hline Spatial[4] & 6 & 7 & 97.6 & 92.9 \\
\hline Spatial & 28 & 4 & 96.4 & 93.8 \\
\hline $\begin{array}{c}\text { Spatio- } \\
\text { Temporal }\end{array}$ & 28 & 4 & 97.3 & 96.4 \\
\hline
\end{tabular}

Table 1: Recognition results on old and new databases

Different cut-off frequencies were used to determine the appropriate number of Fourier components. The results showed that only a small fraction of the components are required to obtain the rates in table 1. For purposes of classification (recognition), the similarity differences between the Fourier descriptions of the gait signatures are then calculated using Euclidean distance, that is

$$
S D_{i, j}=\sum\left(\left|F D_{i}^{\prime}(u, v)\right|-\left|F D_{j}^{\prime}(u, v)\right|\right)
$$

where $S D_{i, j}$ is the similarity difference between gait signatures $i$ and $j$.

\section{4: Recognition by Symmetry}

The database used in this work has 28 subjects each with 4 sequences, giving 112 image sequences. Each image sequence is used once as test data with the remainder as the training data. Having computed the similarity difference between a test subject and the training data, the $k$-nearest neighbour rule is then applied for classification, that is we search for the gait signature in the training that gives the least similarity difference value. Here, we used $k=1$ and $k=3$ and the results are as summarised in Table 1. We include the earlier results on a much smaller database. It is pleasing to see that performance is maintained on the larger database. For $k=1$, the recognition rates of the spatial approach and our new approach were $96.4 \%$ and $97.3 \%$ respectively. The corresponding recognition rates for $k$ $=3$ were $93.8 \%$ and $96.4 \%$. Performance analysis suggests that better classification might arise from feature space classification, or a more sophisticated classifier than the $k$ nearest neighbour rule, but we are 


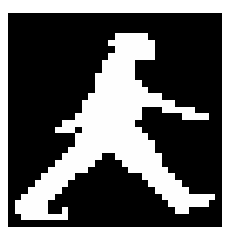

$32 \times 32$

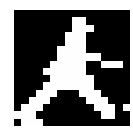

$16 \times 16$
$\mathbf{A}$ $8 \times 8$

Fig. 4: Lower Resolution Images

concerned with more basic issues here. It is clear from the results that the new approach gives a better result for both $k=1$ and $k=3$, emphasising that including time as well as space (body dynamics as well as disposition) is beneficial in symmetry analysis of moving objects.

\begin{tabular}{|c|c|c|c|}
\hline \multirow{2}{*}{ Image size } & \multirow{2}{*}{ \#subj.(\#seq.) } & \multicolumn{2}{|c|}{ Classification rate (\%) } \\
\cline { 3 - 4 } & $28(4)$ & 97.3 & 96.4 \\
\hline $64 \times 64$ & $28(4)$ & 97.3 & 92.9 \\
\hline $32 \times 32$ & 28.3 & 67.0 \\
\hline $16 \times 16$ & $28(4)$ & 84.0 & 33.0 \\
\hline $8 \times 8$ & $28(4)$ & 57.1 & \\
\hline
\end{tabular}

Table 2: Recognition Results for Reduced Resolution

Example images used for low resolution analysis are shown in Fig. 4. Images are usually normalised to $64 \times 64$ to reduce computational demand. If enlarged, the images would highlight the loss of detail at lower resolutions. Reduction in resolution by half to $32 \times 32$ changes recognition performance little for $k=3$. In fact, reduction to $16 \times 16$ is where no other biometric could be perceived, but where recognition by gait still occurs to some extent (one far better than random performance). Again, this suggests that symmetry gives potential for gait as a biometric and for movement analysis in general.

\section{5: Conclusions and Further Work}

The development of symmetry has continued to support the contention that the symmetry of human gait can indeed be used for recognition, as supported by a psychology view and our earlier works on a small database of six subjects. The symmetry operator, essentially, forms an accumulator of points, which are measures of the symmetry between image points to give a symmetry map. This has now been extended to use a spatio-temporal basis, by reformulating the distance metric. Now, recognition is based on body dynamics as well as on body shape. By using the new symmetry operator, the Discrete Fourier Transform and a basic nearest-neighbour approach, the results have produced a recognition rate of over $97 \%$ on a database of 28 subjects. This is a very promising result and further experiments need to be carried out. We have also shown how the new approach can handle lowresolution imagery where other biometrics are obscured. In future we aim to investigate further biometric capabilities, alterations to implementation and consequences of parameterisation, and also potential for exploitation in other applications.

\section{References}

[1] C. BenAbdelkader, R. Cutler, H. Nanda and L. Davis, "EigenGait: Motion-based Recognition using Image Selfsimilarity", $3^{\text {rd }}$ Int. Conf. AVBPA, 2001,pp 284-294.

[2] D. Cunado, M. S. Nixon and J. N. Carter, "Gait extraction and description by evidence gathering", Proc. 2nd Int. Conf AVBPA99, Washington, 1999, pp 43-48.

[3] J. T. Cutting, D. R. Proffitt and L. T. Kozlowski, "A biomechanical invariant for gait perception", J. Exp. Psych.: Human Perception and Perf., 1978, pp 357-372.

[4] J. B. Hayfron-Acquah, M. S. Nixon and J. N. Carter, "Recognising human and animal movement by symmetry", Proc. IEEE ICIP 2001, Greece, pp 290-293.

[5] M. S. Nixon, J. N. Carter, P. S. Huang and S. V. Stevenage, "Automatic gait recognition", In: Biometrics, 11, pp 231-250, Kluwer, 1999

[6] S. A. Niyogi and E. H. Adelson, "Analysing and recognising walking figures in xyt", In: Proc. CVPR, 1994, pp 469-474.

[7] C. J. Parsons and M. S. Nixon, "Introducing focus in the generalised symmetry operator", IEEE Sig. Proc. Lett., 3, 1999, pp 49-51.

[8] D. Reisfeld, H. Wolfson, and Y. Yeshurun, "Context-free attentional operators: the generalised symmetry transform". IJVC, 1995, pp 119-130.

[9] H. Sadeghi, P. Allard, F. Prince, H. Labelle, Symmetry and limb dominance in able bodied gait: a review, Gait and Posture, 12, pp 34-45, 2000

[10] J. D. Shutler, M. S. Nixon and C. J. Harris, "Statistical gait recognition via temporal moments". 4th IEEE SSIAI, 2000, pp 291-295

[11] A. Y. Johnson and A. F. Bobick, "A multi-view method for gait recognition using static body parameters", $3^{\text {rd }}$ Int. Conf. AVBPA, 2001, pp 301-311.

\section{Acknowledgements}

We gratefully acknowledge partial support by the European Research Office of the US Army under Contract No. N68171-01-C-9002. 